$$
\begin{aligned}
& \text { KEK P } 33.100 \\
& \text { sa } 9407
\end{aligned}
$$

KEK Preprint 93-156

November 1993

H

Development and Evaluation of Charge-Sensitive Preamplifier for CsI Calorimeter in the KEK B-factory

M. TANAKA, H. IKEDA, K. TAMAI,

M. TAKEMOTO and H. HAYASHII

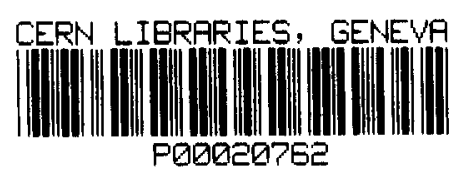

Submitted to the IEEE Nuclear Science Symposium,

San Francisco, CA, U.S.A., Nov. 2-6, 1993. 
National Laboratory for High Energy Physics, 1993

KEK Reports are available from:

Technical Information \& Library

National Laboratory for High Energy Physics

1-1 Oho, Tsukuba-shi

Ibaraki-ken, 305

JAPAN

Phone: 0298-64-1171

Telex: $\quad 3652-534$ (Domestic)

(0)3652-534 (International)

Fax: $\quad 0298-64-4604$

Cable: $\quad$ KEK OHO

E-mail: LIBRARY@JPNKEKVX (Bitnet Address)

library@kekvax.kek.jp (Internet Address) 


\title{
Development and Evaluation of Charge-Sensitive Preamplifier for CsI Calorimeter in the KEK B-factory
}

\author{
Manobu Tanaka, Hirokazu Ikeda, Kunio Tamai, \\ National Laboratory for High Energy Physics, KEK \\ 1-1 Oho, Tsukuba, 305 Japan \\ Megumi Takemoto *and Hisashi Hayashii \\ Nara Women's University \\ Kita-uoya nishi-machi, Nara, 630 Japan
}

\begin{abstract}
We developed a charge-sensitive preamplifier for the electromagnetic calorimeter (CsI(Tl)) for the KEK B-factory. We examined not only noise characteristic and dynamic range but also the stability of the preamplifier. In order to study the stability of the preamplifier, the input/output impedance and the power-supply ripple-rejection ratio were measured for various loads at the input/output node.
\end{abstract}

\section{Introduction}

In the KEK B-factory the barrel part of the electromagnetic calorimeter comprises $7000 \mathrm{CsI}(\mathrm{TI})$ crystals. Each crystal has two photodiodes (HAMAMATSU S2744-03) and two preamplifiers. The capacitance of the photodiode is $100 \mathrm{pF}$ at a bias voltage of $60 \mathrm{~V}$ and the leakage current is less than $4 \mathrm{nA}$. Since a photodiode-readout has several advantages as a photon sensor over phototubes [1], a photodiode has been used for $\operatorname{CsI}(\mathrm{Tl})$ calorimeters[2],[3],[4] and for $B G O,[5],[6]$, where charge-sensitive preamplifiers were commonly employed. In the B-factory experiment we measure the energy of photons and electrons over an energy range between $10 \mathrm{MeV}$ and $8 \mathrm{GeV}[7]$.

At the low-energy region (i.e. below $0.1 \mathrm{GeV}$ ), in order to maintain an energy resolution of better than $1.3 \% / \sqrt{E}$ we must maintain an equivalent noise charge below 700 electrons for a detector capacitance of $100 \mathrm{pF}[7]$. In order to achieve this requirement several JFETs were examined for transconductance and $1 /$ f noise using a Quanteck Semiconductor Noise Analyser. Futhermore, we confirmed the noise characteristics of selected JFETs by a test circuit board of a preamplifier. Details were described in [8]. Since we had to mount two channels in a space of $5 \times 5 \times 2 \mathrm{~cm}^{3}$, it was desirable that the input JFET for the preamplifier was a mini-mold type package. We tested 2SK322, 2SK613,2SK1576, 2SK980 and 2SK932. The JFETs 2 SK 322 and 2 SK613 showed a similar good performance. Though 2 SK 322 have been used for TRISTAN experiment[9], we chose $2 \mathrm{SK} 613$ for the input device because it was difficult to obtain a stable supply of 2 SK 322 .
Since we chose the feedback capacitance of $1 \mathrm{pF}$ for the preamplifier, the output voltage of the preamplifier was about $3 \mathrm{~V}$ for an incident energy of $8 \mathrm{GeV}$. We chose the decay time of $50 \mu \mathrm{sec}$ for the preamplifier. The dynamic range of the preamplifier was specified as $10 \mathrm{~V}$ to be large enough for our application.

We have to consider the stability of the preamplifier circuit. Real parts of the input and output impedance must keep positive for any loading conditions. Since ripples on power rails are main reason of external noise, it is desirable that the power-supply ripple-rejection ratio is high.

We describe a circuit configuration of the preamplifier in section 2 and the performance in section 3 . We summarized this paper in section 4 .

\section{Circuit configuration}

Figure 1 shows the circuit configuration of the preamplifier. $\mathrm{V}_{C C}$ and $\mathrm{V}_{E E}$ are $15 \mathrm{~V}$ and $-5 \mathrm{~V}$. The charge gain is 1 $\mathrm{V} / \mathrm{pC}$, and the power consumption is $120 \mathrm{~mW}$. The circuit is realized by a hybrid circuit on a four-layered printedcircuit board. We chose to use parallel-plate capacitors for $\mathrm{C} 1$ and $\mathrm{C} 2$ using small area or the hybrid circuit. A corner frequency of Q1, at which the device-intrinsic white-noise and the $1 / \mathrm{f}$ noise contributed equally to the noise, was 1 $\mathrm{kHz}$.

While the preamplifier's topology is similar to the previous work published in [10], we put some new features to our circuit. When ripples are fed into power rails of the preamplifier, the ripples are transferred by Q4, Q6, Q7 and Q8 to OUT-E and OUT-C. In order to suppress the effect we have to achieve a high power-supply ripple-rejection ratio (PSRR). In traditional way a passive filter have been used for filtering on power rails of a preamplifier. If we used a resistance of small value and a chip-type capacitor, it would be difficult to achieve a low cutoff frequency of the filter (i.e. lower than a few $\mathrm{kHz}$.). We added filters on $\mathrm{V}_{C C}$ and $\mathrm{V}_{E E}$, which comprised $\mathrm{Q} 5, \mathrm{C} 5, \mathrm{C} 7, \mathrm{R} 8$ and $\mathrm{Q} 9, \mathrm{C} 6, \mathrm{C} 8, \mathrm{R} 7$. If current gains of Q5 and Q9 is large, the cutoff frequency of the filter is determined by time constants of C7,R8 and $\mathrm{R} 7, \mathrm{C} 8$. Since we set the cutoff frequency of $16 \mathrm{~Hz}$, the PSRR became high.

"present address Mitsubishi Co. Itd. 


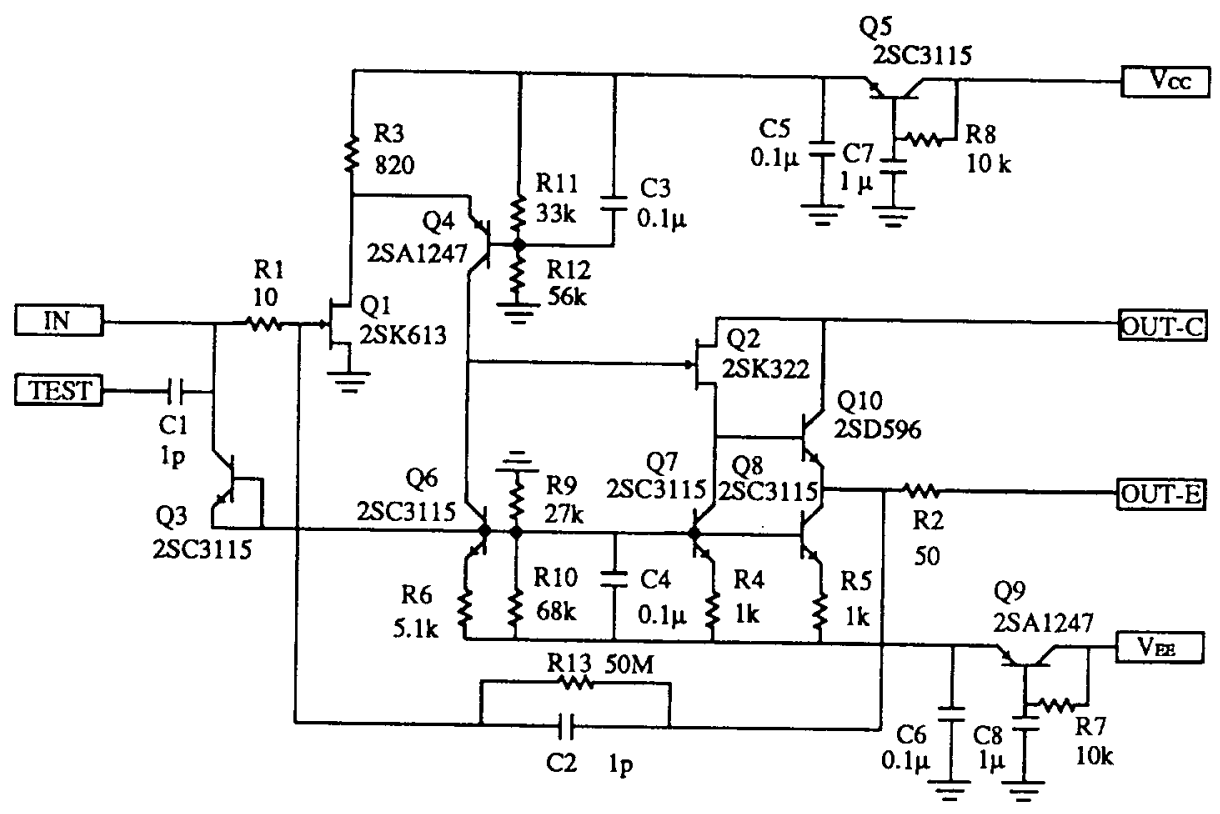

Figure 1. Circuit configuration of the preamplifier.

The output stage of the preamplifier is a Darlington combination of Q2 and Q10. We chose a JFET as the device of Q2 in order to avoid a degradation of the open loop gain for large signal output. The preamplifier has OUT-C and OUT-E so as to drive a transmission line from either the emitter node or the collector node of the preamplifier. We are going to use OUT-C as a signal output-node.

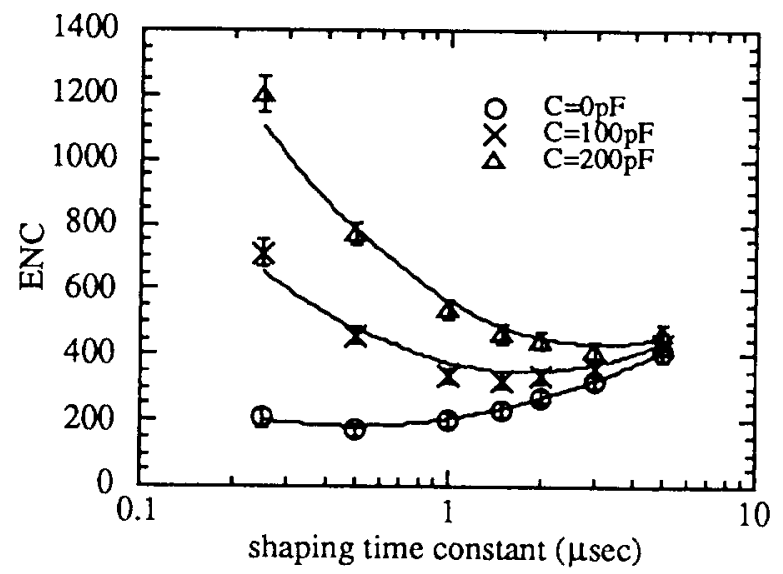

Figure 2. Time constant dependence of the equivalent noise charge. The solid lines are results of the fitting.

\section{Performance}

\subsection{Noise}

We measured the equivalent noise charge(ENC) for various detector capacitances $\left(\mathrm{C}_{d}\right)$ and various shaping times using a pulse generator (HP8110A), a shaper (ORTEC 450 Research Amplifier) and an oscilloscope (Tektronix
11403A). A test pulse was fed into TEST, and we added $\mathrm{C}_{d}$ at IN. OUT-C was pulled up by $1 \mathrm{k} \Omega$ to $\mathrm{V}_{C C}$. The output signal from OUT-C was terminated at the input of the shaper by $50 \Omega$ after an AC-coupling capacitor. The shaping function of the shaper was described in [11]. We chose the integration time and the differentiation time of the shaper to be equal. Figure 2 shows ENC as a function of the shaping time constant. The ENC is optimized at the time constant of around $1.5 \mu \mathrm{sec}$ for $\mathrm{C}_{d}=100 \mathrm{pF}$. Since the ENC is less than 400 electrons at the detector capacitance of around $100 \mathrm{pF}$, the preamplifier satisfies our requirement for ENC.

In order to understand the behaviour of ENC we fitted the measurement values by a theoretical equation[12]. Since a contribution of $1 / \mathrm{f}$ noise of about 36 electrons at $\mathrm{C}_{d}=100 \mathrm{pF}$ for $\mathrm{CR}(\mathrm{RC})^{2}$ shaping, we considered the part of the $1 / \mathrm{f}$ noise for the fitting. The fitting parameters were stray capacitance $\left(\mathrm{C}_{s}\right)$, the transconductance $\left(\mathrm{g}_{m}\right)$ and the leakage current $\left(i_{\text {leak }}\right)$ at the IN node. We assumed the junction capacitance of Q1 was $6.6 \mathrm{pF}$ from a data sheet. The filtering function of the shaper was approximated to a Poisson filter(CR(RC) $)^{2}$ ). An error from the approximation was $\pm 2 \%$. The fitting results were $\mathrm{C}_{s}=(30 \pm 6.8) \mathrm{pF}$, $\mathrm{g}_{m}=(20 \pm 2.5) \mathrm{mS}$ and $i_{\text {leak }}=\left(0.0 \pm 1.9 \times 10^{-2}\right) \mathrm{nA}$. We measured the stray capacitance of the test bench and the printed-circuit board by a C-V analyzer(KEITHLEY 590). Transistors connected IN node(Q1 and Q3) were removed from the printed-circuit board when we measured the stray capacitance. The stray capacitance was $15 \mathrm{pF}$ on the test bench, and $10 \mathrm{pF}$ on the printed-circuit board of the preamplifier. The stray capacitance on the printedcircuit board contributes to the total detector capacitance by $10 \%$. We could reduce the stray capacitance by changing the layout pattern on the circuit board. 


\subsection{Dynamic range}

We measured the integral nonlinearity at OUT-E node and the OUT-C node by an oscilloscope (Tektronix $11403 \mathrm{~A}$ ). A test pulse was fed into TEST of the preamplifier. The biasing condition of the preamplifier was the same as that for the noise measurement. The preamplifier drove a $50 \Omega$ transmission cable, and the cable was terminated at the input of the oscilloscope. Figure 3 shows the integral nonlinearity of the preamplifier at OUT-E and OUT-C. The output voltage is $5 \mathrm{~V}$ at OUT-E for $10 \mathrm{~V}$ test pulse because of the back termination of R2. The output voltage is $10 \mathrm{~V}$ at OUT-C for an input test pulse of $10 \mathrm{~V}$. Both integral nonlinearities are less than $0.3 \%$ for the test pulse amplitude of $10 \mathrm{~V}$.

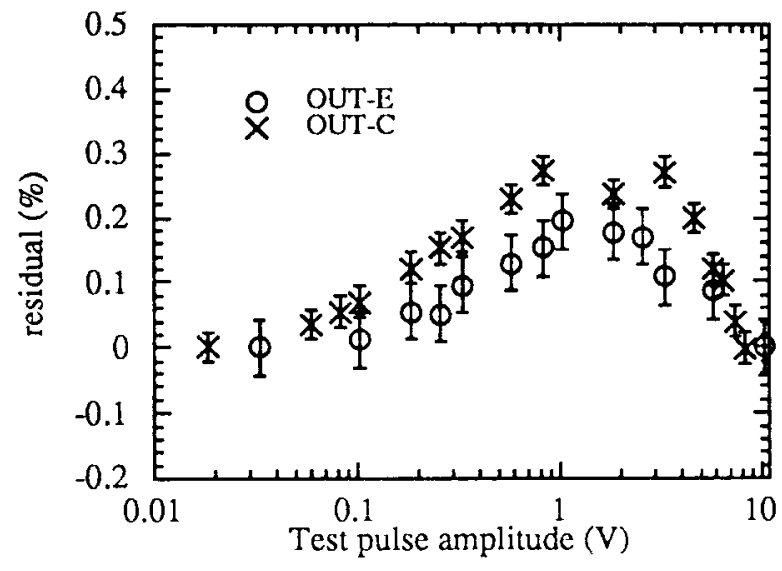

Figure 3. Integral nonlinearity.

\subsection{Impedance}

The input and output impedances were measured by a network analyzer (ADVANTEST R4611) together with a SWR bridge. Figure 4 shows the real part of the input impedance as a function of the frequency.

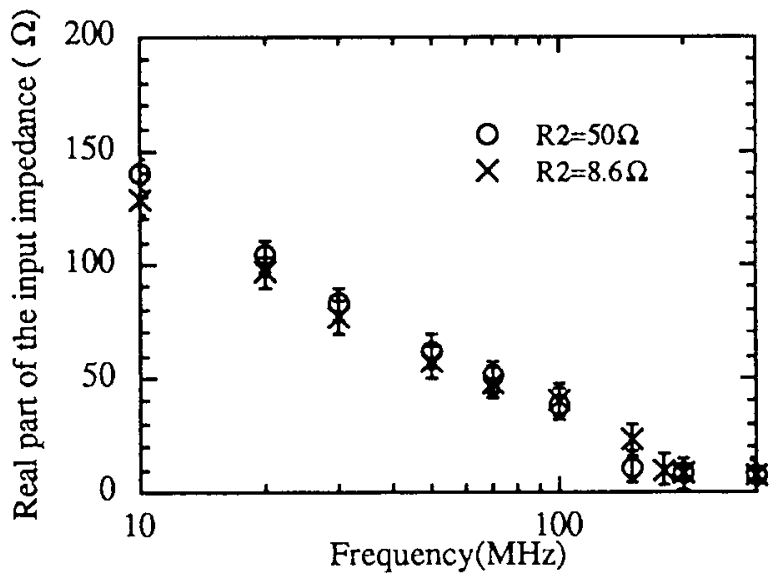

Figure 4. Frequency dependence of the input impedance.

The input impedance was measured at $\mathrm{R} 2=8.6$ and 51 $\Omega$. The real part of the input impedance was positive in the full scale range of the measurement system (i.e. 10
$\mathrm{MHz} \sim 300 \mathrm{MHz}$.$) . The SWR bridge limited the lower$ side of the range, and the upper limit came from the network analyzer. Figure 5 shows the real part of the output impedance. We measured the output impedance for $C_{d}=0$, 51 and $100 \mathrm{pF}$. The real part of the output impedance was maintained larger than $51 \Omega$. We confirmed stable operation of the preamplifier with a photodiode of $\mathrm{C}_{d}=100 \mathrm{pF}$.

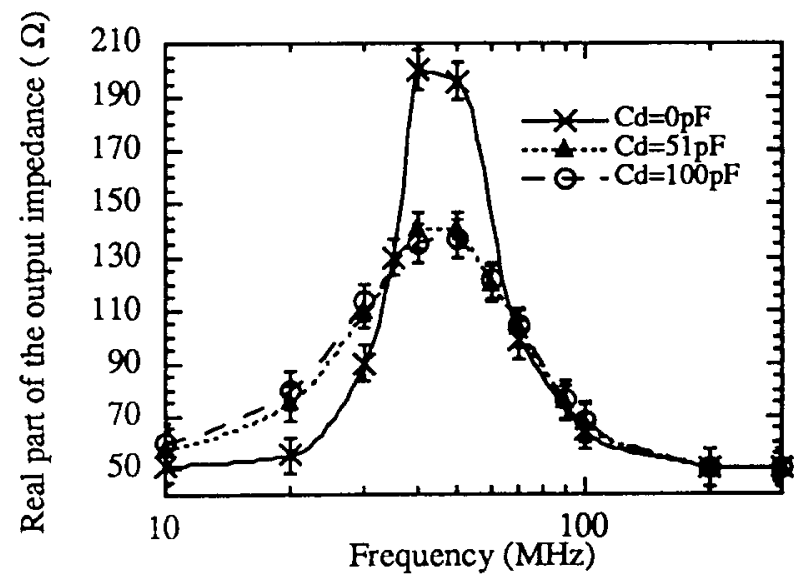

Figure 5. Frequency dependence of the output impedance. The solid line, the dashed line and the dotted line are guide to the eyes.

\subsection{PSRR}

We measured PSRRs for $\mathrm{V}_{C C}$ and $\mathrm{V}_{E E}$ at OUT-C and OUT-E. Figure 6 shows the setup when we measured PSRR at OUT-C for $\mathrm{V}_{C C}$. When we measured PSRR at OUT-E, OUT-C node was terminated by $51 \Omega$ via a capacitor of $50 \mu \mathrm{F}$. $V_{E E}$ was supplied from the output node of LH0032 when we measured PSRR for $\mathrm{V}_{E E}$. The input power from the network analyser was $-10 \mathrm{dBm}$. Figures 7 (a) and (b) show PSRR of $V_{C C}$ as a function of the frequency and that of $\mathrm{V}_{E E}$. We added $\mathrm{C}_{d}$ of $100 \mathrm{pF}$ at IN in the measurement. PSRR for $V_{C C}$ and for $V_{E E}$ was less than $-60 \mathrm{~dB}$ around $1 \mathrm{MHz}$.

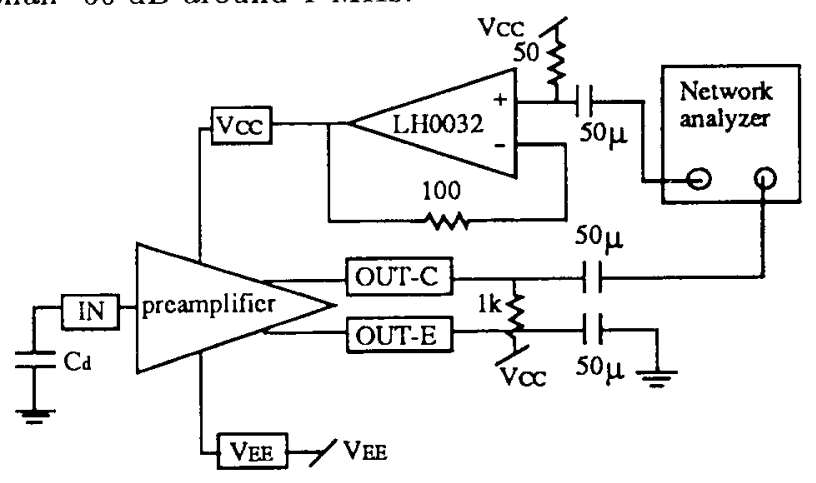

Figure 6. Test-bench set up for a PSRR measurement for $\mathrm{V}_{C C}$.

Figure 8 shows PSRR as a function of detector capacitance. We measured PSRR at $2 \mathrm{kHz}$ and $1 \mathrm{MHz}$ for $\mathrm{C}_{d}=0$, $10,51,100$ and $200 \mathrm{pF}$. PSRR at $2 \mathrm{kHz}$ was saturated 
above $51 \mathrm{pF}$, and there was no capacitance dependence of PSRR at $1 \mathrm{MHz}$. We can achieve a PSRR of $-40 \mathrm{~dB}$ at 2 $\mathrm{kHz}$ and $-60 \mathrm{~dB}$ around $1 \mathrm{MHz}$. This high PSRR is enough for our application.

\section{Summary}

A charge-sensitive preamplifier was developed for the CsI $(\mathrm{Tl})$ calorimeter. The preamplifier was examined for noise, dynamic range, input impedance, output impedance, and power supply ripple rejection ratio. The equivalent noise charge was less than 400 electrons for a detector capacitance of $100 \mathrm{pF}$. The optimum time constant of the shaper (ORTEC 450) was about $1.5 \mu \mathrm{sec}$. The integral nonlinearity of the preamplifier was less than $0.3 \%$ for maximum output voltage of $10 \mathrm{~V}$. We measured the input and output impedances of the preamplifier. The preamplifier worked stably for a detector capacitance of $100 \mathrm{pF}$. We measured the power supply ripple rejection ratio for $V_{C C}$ and $V_{E E}$. We were able to achieve a PSRR of $-40 \mathrm{~dB}$ at $2 \mathrm{kHz}$ and a PSRR of less than $-60 \mathrm{~dB}$ around $1 \mathrm{MHz}$.
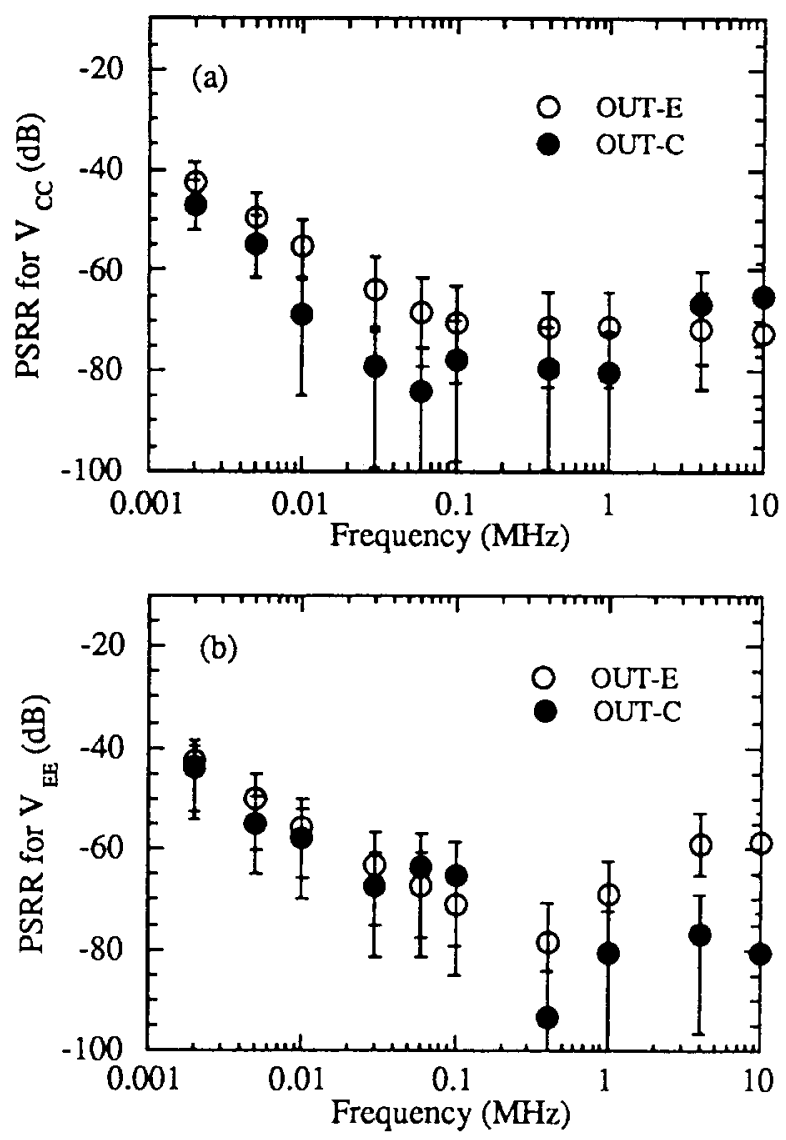

Figure 7. Frequency dependence of PSRR. (a) shows PSRR for $V_{C C}$. (b) shows PSRR for $V_{E E}$.

\section{Acknowledgements}

We thank K. Tanimoto, H. Sawachi of Meisei Electronic Co. Itd, M. Fukushima, other members of the CsI calorimeter group and T. Matsushita for their support. We also appreciate the discussions with $\mathrm{H}$. Kaneda, $\mathrm{T}$. Tamura and T. Murakami. We would like to express our appreciation for the encouragement of directors, S. Iwata, F. Takasaki, M. Kobayashi and K. Nakai.

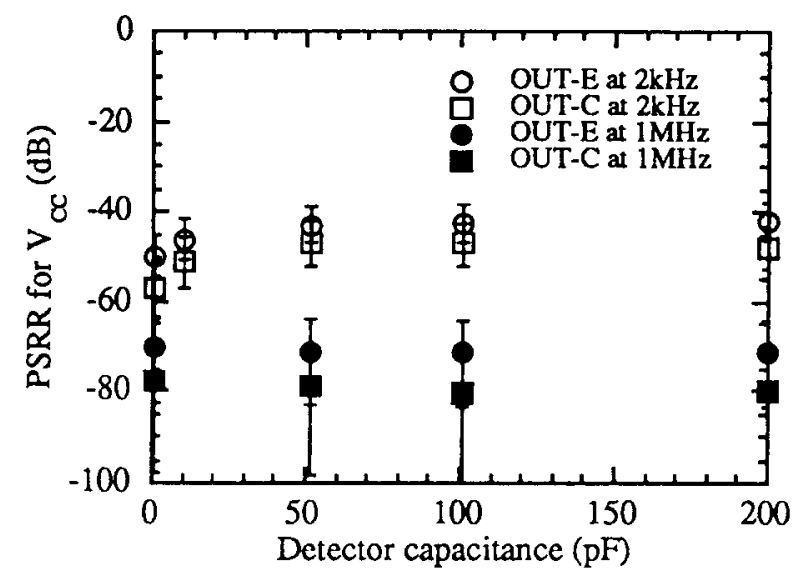

Figure 8. Detector capacitance dependence of the PSRR for $V_{C C}$.

\section{References}

[1] B. Grassmann et al., Nucl. Inst. \& Meth. $228(1985) 323$.

[2] Z. Bian et al., Nucl. Inst. \& Meth. A239(1985)518.

[3] M. Suffert et al., 7th International workshop on room temperature semiconductor X- $\gamma$-ray detectors and associated electronics, 23-28 September 1991, Ravello, Italy.

[4] N. Sato et al., Society of Photo-Optical Instrumentation Engineering, vol 1734, Gamma-ray detectors, July 1992, San Diego, USA.

[5] M. Goyot et al., Nucl. Inst. \& Meth. A263(1988)180.

[6] H. Hayashii et al., Nucl. Inst. \& Meth. A316(1992)202.

[7] B-Physics Task Force, KEK Report 93-1, April 1993

[8] M. Takemoto, Master thesis (in Japanese), Nara Women's University, March 1993

[9] H. Ikeda et al., IEEE Trans. on NS-34(1987)191

[10] D. A. Landis et al., IEEE Trans. on NS-29(1982)573

[11] EG\&G ORTEC Model 450 Research Amplifier Operating and Service Manual Rev 47, 1992.

[12] V. Radeka, Ann. Rev. Nucl. Part. Sci. 38(1988)217 